\title{
Developing controllable hypermutable Clostridium cells through manipulating its methyl-directed mismatch repair system
}

\author{
Guodong Luan ${ }^{1,2}$, Zhen Cai ${ }^{1 凶}$, Fuyu Gong ${ }^{1,2}$, Hongjun Dong ${ }^{1}$, Zhao Lin ${ }^{1,2}$, Yanping Zhang ${ }^{1}$, Yin Li ${ }^{1 凶}$ \\ ${ }^{1}$ CAS Key Laboratory of Microbial Physiological and Metabolic Engineering, Institute of Microbiology, Chinese Academy of \\ Sciences, Beijing 100101, China \\ ${ }^{2}$ University of Chinese Academy of Sciences, Beijing 100049, China \\ $\triangle$ Correspondence: caiz@im.ac.cn (Z. Cai), yli@im.ac.cn (Y. Li)
}

Received September 5, 2013 Accepted September 25, 2013

\begin{abstract}
Development of controllable hypermutable cells can greatly benefit understanding and harnessing microbial evolution. However, there have not been any similar systems developed for Clostridium, an important bacterial genus. Here we report a novel two-step strategy for developing controllable hypermutable cells of Clostridium acetobutylicum, an important and representative industrial strain. Firstly, the mutS/L operon essential for methyldirected mismatch repair (MMR) activity was inactivated from the genome of $C$. acetobutylicum to generate hypermutable cells with over $\mathbf{2 5 0}$-fold increased mutation rates. Secondly, a proofreading control system carrying an inducibly expressed mutS/L operon was constructed. The hypermutable cells and the proofreading control system were integrated to form a controllable hypermutable system SMBMutC, of which the mutation rates can be regulated by the concentration of anhydrotetracycline (aTc). Duplication of the miniPthl-tetR module of the proofreading control system further significantly expanded the regulatory space of the mutation rates, demonstrating hypermutable Clostridium cells with controllable mutation rates are generated. The developed $C$. acetobutylicum strain SMBMutC2 showed higher survival capacities than the control strain facing butanol-stress, indicating greatly increased evolvability and adaptability of the controllable hypermutable cells under environmental challenges.
\end{abstract}

KEYWORDS Clostridium acetobutylicum, mutation rates, hypermutable cells, artificial control

\section{INTRODUCTION}

Microbial evolution is promoted by genetic modifications on genomic architectures and compositions (Hastings et al., 2000; Matic et al., 2004; Conrad et al., 2009). Spontaneous mutations during DNA replication provide an important evolutionary force for microbes encountering environmental fluctuations, and accelerated accumulation of adaptive mutations can be expected to confer survival and growth advantages on individual cell level as well as on population level (Taddei et al., 1997; Tenaillon et al., 1999). Thus, hypermutable cells with elevated mutation rates play an important role for microbial adaptation and evolution under environmental stresses, e.g. rapid emergence of bacterial resistance to multi antibiotics (Tanabe et al., 1999; Perron et al., 2010). Recently hypermutable cells have also shown great potential in biotechnological applications, based on the ability of increasing the genetic variability at the genome level (Greener et al., 1997; Selifonova et al., 2001). However, the percentage of the hypermutable cells in microbial populations is generally low, and microbial cells tend to keep the mutation rates of genome replication at a relatively low level to avoid accumulation of deleterious spontaneous mutations (Kimura, 1967; Ishii et al., 1989; Kondrashov, 1995). Usually, the accuracy of genome replication is guaranteed by multiple and hierarchal mechanisms. This mainly includes base selection, $3^{\prime}->5$ ' exonuclease, and MMR (Methyl-directed Mismatch Repair) (Echols and Goodman, 1991; Kunkel and Bebenek, 2000). For better understanding and harnessing microbial evolution and genome plasticity, the natural quality control system of genome replication should be disturbed and replaced with artificial control mechanism, by which mutation rates of genome replication can be controlled and regulated as 
requirement.

Clostridium is the second largest bacteria genera in size, and classified as Gram-positive endospore-forming obligate anaerobes (Andreesen, 1989; Rehner and Samuels, 1994). A large portion of Clostridium species, e.g. C. botulinum, C. tetani, C. septicum, and C. difficile pose serious threats to human health (Sakaguchi, 1982; Kennedy et al., 2005; Bartlett, 2006). On the other hand, many other Clostridium species, e.g. C. acetobutylicum, C. thermocellum, and C. bifermentans, show great significance on biotechnological application for solvents production and pollutant biodegradation (Jones and Woods, 1986; Lewis et al., 1996). Understanding and manipulating physiological and behavioral mechanisms of such Clostridium species requires better controllability of the microbial evolution, which can be achieved by construction of a controllable hypermutable system in Clostridium.

Previously, artificial regulation of cellular mutation rates was achieved by introducing proofreading-deficient DNA polymerase mutants (Selifonova et al., 2001; Shimoda et al., 2006; Abe et al., 2009) or inactivation of the nonessential proofreading mechanism (Sasaki et al., 2000; Emlyn-Jones et al., 2003), through which the natural quality-control mechanism for DNA replication can be disturbed. To date, this strategy has been applied in several model microbes, including Escherichia coli, Bacillus subtilis, and Saccharomyces cerevisiae (Selifonova et al., 2001; Emlyn-Jones et al., 2003; Endo et al., 2006; Shimoda et al., 2006), and the alteration of mutation rates is usually determined by a specific mutator gene, resulting in a single-switch control pattern. We aimed to design a novel and universal method through which microbial mutation rates can be rapidly regulated to diverse levels. To this end, we proposed a two-step strategy for construction of an artificial control of microbial mutation rates. The first step is to disrupt the natural cellular mechanisms for controlling the mutation rates by inactivating the relevant genes, generating cells with significantly increased mutation rates (hypermutable cells). The second step is to construct a proofreading control system carrying the genes that have been inactivated in the hypermutable cells, under the control of an inducible promoter. Combination of the hypermutable cells with the controlled expression of relevant genes is expected to generate an artificial control of mutation rates.

Using this concept, we constructed a controllable hypermutable system in Clostridium acetobutylicum, which is a representative species of the genus Clostridium with industrial relevance. By identification and deactivation of the chromosomally encoded mutS/L operon, we obtained a hypermutable cell SMBMut5, with over 250 -fold increased mutation rates achieved. Subsequently, a proofreading control system bearing an anhydrotetracycline (aTc)-inducible mutS/L expression system was then constructed and introduced into SMBMut5, generating controllable hypermutable systems SMBMutC and SMBMutC2, in which the mutation rates of genome replication can be well controlled and regulated by the concentrations of exogenous aTc molecules. Facing butanol-stress, cells of SMBMutC2 under the evolving state showed much higher sur- vival rate than the wildtype control, indicating that evolvability and adaptability of the controllable hypermutable Clostridium cells developed in this work were greatly increased. The newly developed controllable hypermutable system enables better harnessing of genome replication mutation rates of $C$. acetobutylicum, and will encourage the development of similar systems in other Clostridium species.

\section{RESULTS}

\section{Design of the controllable hypermutable system in C. acetobutylicum}

To achieve artificial control of genome replication mutation rates in $C$. acetobutylicum, we designed a controllable hypermutable system consisted of a hypermutable cell and a proofreading control system, as shown in Fig. 1A. For construction of the hypermutable cell, the chromosomally located mutS and mutL on genome of $C$. acetobutylicum were knocked out. Since mutS and mutL are coding for activities of MMR, an important proofreading mechanism for DNA replication (Echols and Goodman, 1991; Kunkel and Bebenek, 2000; Kunkel, 2004), the resulted mutants are expected to exhibit significantly increased mutation rates due to decreased fidelity of genome replication. With regards to introduction of a proofreading control system, the mutS/L operon was placed under the control of an aTc inducible promoter that we developed previously for Clostridium (Dong et al., 2012). The hypermutable cell containing the proofreading control system thus forms a system that is capable of artificially controlling the mutation rate of genome replication. In the absence of aTc, the promoter is bound by TetR to block the expression of mutS and mutL, meaning the MMR function is deficient so as the mutation rate is elevated to a maximal level of the hypermutable state. In the presence of aTc, the TetR repressor is bound by aTc, thus the promoter will be induced to initiate the expression of mutS and mutL, ensuring a high fidelity of genome replication. As the expression strength is dependent on aTc concentration, the mutation rate can be gradually recovered to a regular level as aTc concentration increases (Fig. 1B). When aTc concentration achieved an optimal value for mutS/L expression, mutation rates will be regulated to the minimal level of the system. In summary, the switch ON/OFF and fine-tuning of the hypermutable state can be achieved in the absence or presence of an appropriate concentration of aTc.

Generation of hypermutable cells of $C$. acetobutylicum by inactivation of mutS/L operon

To create hypermutable cells of $C$. acetobutylicum, we aimed to inactivate the MMR-system-encoding genes. C. acetobutylicum SMB009 is a derivative of C. acetobutylicum DSM1731, of which the complete genome has been sequenced (Bao et al., 2011). Chromosomally located SMB_G1862 and SMB_ G1861 genes were annotated as mutS and mutL, respectively. ClosTron method was then applied to inactivate these two 


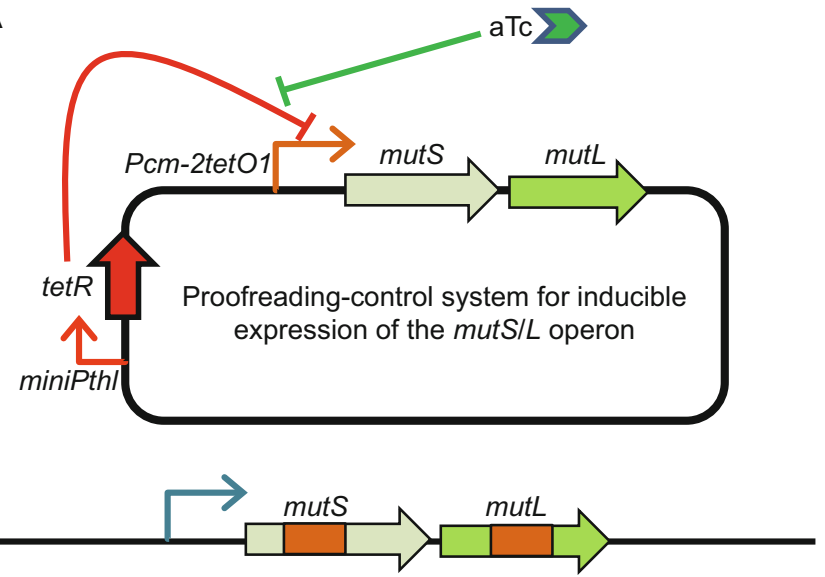

Intron-disrupted mutS/L operon on chromosome of hypermutable C. acetobutylicum cells

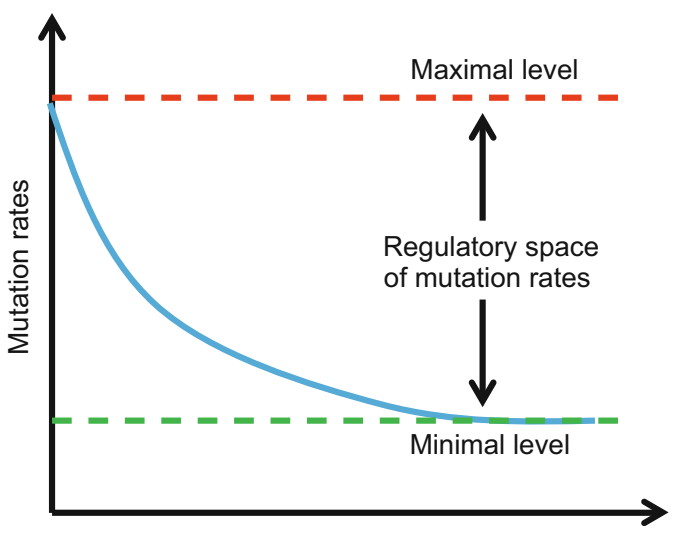

aTc concentrations

Figure 1. Design of the controllable hypermutable system in C. acetobutylicum. (A) Chromosome located mutS and mutL of C. acetobutylicum were disrupted by intron. An exogenous vector carrying mutS/L operon under control of an aTc inducible promoter Pcm-2tetO1 was introduced into the cell as a proofreading-control system. In the absence of aTc, the TetR molecules bound to the Pcm$2 t e t O 1$ promoter and inhibited expression of $m u t S / L$, leading to an elevation of mutation rates. In the presence of aTc, the TetR molecules were bound by aTc, and the expression of $m u t S / L$ was activated, leading to an increased fidelity of an increased fidelity of genome replication. (B) Mutation rates of the controllable hypermutable system in C. acetobutylicum can be well controlled in a regulatory space by regulation of aTc concentrations. When aTc was completely absent, mutation rates achieved a maximal level due to the top level of TetR inhibition. When aTc concentration achieved an optimal value for mutS/L expression, mutation rates were regulated to the minimal level for the system.

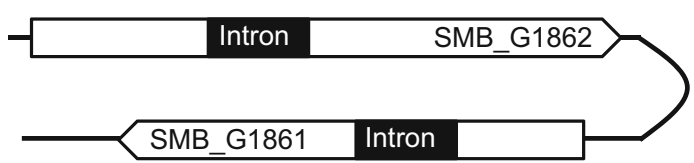

B

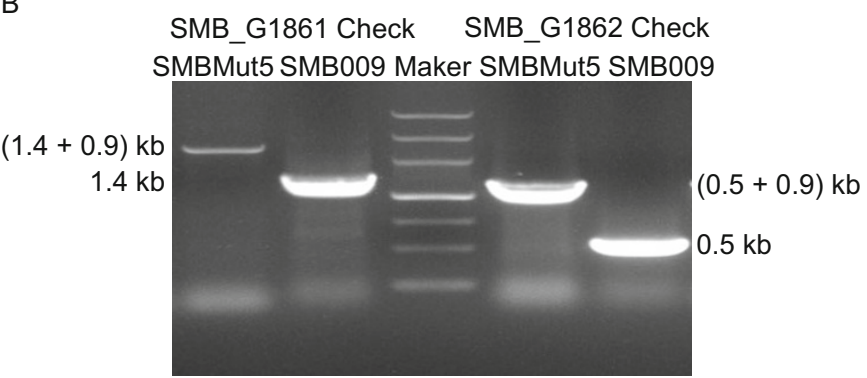

C

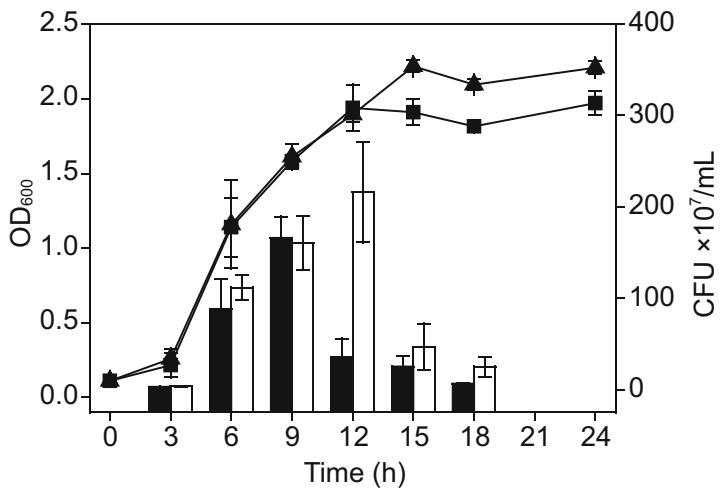

Figure 2. Construction and evaluation of mutS/L disrupted strain SMBMut5. (A) Schematic show of the disruption pattern of mutS/L operon on the genome. Two introns were separately inserted into the CDS of SMB_G1862 gene and SMB_G1861 gene for completely inactivation of mutS/L operon. (B) Identification of the SMBMut5 and SMB009 strains by primers mutS-disruption-check-F/R (the right two lanes) and mutL-disruption-check-F/R (the left two lanes). (C) Growth assay of SMBMut5 and SMB009 strain. OD 600 value (points and lines) and CFU counts (bars) were measured during the growth of SMBMut5 (filed triangles and white bars) and SMB009 strains (filed squares and black bars) in $24 \mathrm{~h}$.

genes (Fig. 2A), generating mutS/mutL single mutant or mutSmutL double mutant (Table 1). Insertions of the ClosTron into the target gene sites were verified via PCR amplifications (Fig. 2B) and subsequent sequencing of PCR products.

To confirm whether the inactivation of mutS/L operon led to increase of mutation rates in C. acetobutylicum, we determined the frequencies of generating rifamycin-resistant (Rif25R) colonies, which were commonly used to reflect microbial mutation rates (Sasaki et al., 2000; Selifonova et al., 2001; EmlynJones et al., 2003). As expected, inactivation of $m u t S / L$ operon 
Table 1. Genotypes and mutation rates evaluation of the series of mutS/L inactivated strains

\begin{tabular}{llll}
\hline Strain & Relative genotypes $^{\text {a }}$ & Mutation rates $^{\mathrm{b}}$ & Relative mutation rates $^{\mathrm{c}}$ \\
\hline SMB009 & & $(0.0056 \pm 0.0003) \times 10^{-6}$ & 1 \\
SMBMut2 & mutL::intron & $(0.83 \pm 0.02) \times 10^{-6}$ & 148.8 \\
SMBMut3 & mutS::intron & $(0.87 \pm 0.17) \times 10^{-6}$ & 155.4 \\
SMBMut5 & mutS::intron, mutL::intron & $(1.44 \pm 0.16) \times 10^{-6}$ & 257.9 \\
\hline${ }^{\text {a }}$ All of the SMBMut2, SMBMut3, and SMBMut5 strains were obtained from SMB009 by genetic modifica- \\
tion with ClosTron gene inactivation approach. \\
${ }^{b}$ Mutation rates data were calculated from both the total numbers of live cells and Rif25 resistant cells. To \\
calculate the standard deviation, every experiment was repeated at least 3 times. \\
${ }^{\mathrm{C}}$ Relative mutation rates were obtained by comparison with that of the SMB009 as 1.
\end{tabular}

significantly increased the mutation rates (Table 1). The mutSmutL disrupted strain SMBMut5 showed an over 250-fold increased frequency for generating Rif25R colonies. In addition, the mutL-disrupted strain SMBMut2 and mutS-disrupted strain SMBMut3 also showed an approximately 150-fold increased mutation rate, respectively, comparing to that of the wildtype control C. acetobutylicum SMB009 (Table 1). Determination of the mutation rates indicated that inactivation of the mutS/L operon disturbed the quality control system for ensuring the high fidelity of genome replication in $C$. acetobutylicum, thus leading to accumulation of significantly increased spontaneous mutations.

Further analysis revealed that inactivation of the mutS/L operon did not inhibit the growth of $C$. acetobutylicum. The SMBMut5 strain showed a similar but prolonged growth curve with the control (Fig. 2C). Both the optical density and the live cells of strain SMBMut5 in culture broth kept increasing for a longer process than that of SMB009, possibly due to the enhanced adaptability caused by high mutation rates under complex and tough conditions when the culture entered stationary phase. Thus $C$. acetobutylicum SMBMut5 was selected as the hypermutable cell for further investigation.

\section{Assembling and evaluation of the controllable hypermutable system in C. acetobutylicum}

To artificially control the mutation rate of the hypermutable cells of $C$. acetobutylicum, we cloned the mutS/L operon from C. acetobutylicum DSM1731 and inserted it into a previously developed inducible-gene-expression system in C. acetobutylicum, pGusA2-2tetO1 (Dong et al., 2012). The new plasmid was termed as pMutS/L-2tetO1, in which the expression of mutS/L was controlled by an aTc inducible promoter. Plasmid pMutS/L-2tetO1 was transformed into strain SMBMut5, generating a new strain designed as SMBMutC (Fig. 3A). For evaluation and analysis, strain SMB009 carrying pMutS/L-2tetO1, designed as SMB9M, was used as a control.

The hypothesis is that strain SMBMutC generated by combination of the hypermutable cell with the proofreading control system should work as a controllable hypermutable system. To verify this, we determined the mutation rates of strains SMBMutC and SMB9M under serial concentrations of aTc. As shown in Fig. 3B, the mutation rate of SMBMutC is responsive to the increased concentrations of aTc. In the absence of aTc, strain SMBMutC showed an elevated mutation rates $(0.35 \times$ $\left.10^{-6}\right)$, 95-fold and 62-fold higher than that of strain SMB9M $\left(0.0037 \times 10^{-6}\right)$ and SMB009 $\left(0.0056 \times 10^{-6}\right)$, respectively. When an increased concentration aTc was added to the medium, the mutation rates of strain SMBMutC were gradually decreased. When the aTc concentrations reached $50 \mu \mathrm{g} / \mathrm{mL}$, the mutation rates of SMBMutC reverted to a regular level $(0.0063$ $\times 10^{-6}$ ) comparable to that of the controls.

Duplication of the tetR module optimized the controllability of the controllable hypermutable system

As shown in Table 1 and Fig. 3B, introduction of pMutS/L-2tetO1 into strain SMBMut5 led to a 4-fold decrease of background mutation rates in the absence of aTc (from $1.44 \times 10^{-6}$ to 0.35 $\times 10^{-6}$ ), indicating leaky expression of mutS/L occurs. To increase the capability of the controllable hypermutable system for regulating the mutation rate, we aimed to increase the stringency of the inducible mutS/L expression system. Since the tetR gene in pMutS/L-2tetO1 encodes a tetR protein, which recognizes and combines with the 2 tetO1 region in the promoter, we hypothesized that increasing the gene dose of tetR might reduce leaky expression, thus improving the stringency of the system. We therefore duplicated the miniPthl-tetR module on pMutS/L-2tetO1 and obtained the new plasmid, which is designed as pMutS/L-2tetO1-2tetR.

We introduced the newly constructed pMutS/L-2tetO12tetR into strain SMBMut5 and obtained strain SMBMutc2 (Fig. 4A). Mutation rates determination (Fig. 4B) revealed that strain SMBMutC2 showed a better controllability than that of SMBMutC. In the absence of aTc, background mutation rates of SMBMutC2 $\left(0.71 \times 10^{-6}\right)$ is 2-fold higher than that of SMBMutC $\left(0.35 \times 10^{-6}\right)$, suggesting the leaky expression of $m u t S / L$ was reduced in the new system. Further analysis revealed that the mutation rate of strain SMBMutC2 could also be well regulated by aTc concentrations. In the aTc concentration of $0,50,100$, and $200 \mu \mathrm{g} / \mathrm{mL}$, mutation rates of strain SMBMutC2 can be increased by 120 -fold, 40-fold, 10-fold, and 3-fold respectively, comparing with that of the wildtype control SMB009, ranging over 3 orders of magnitudes. 
A

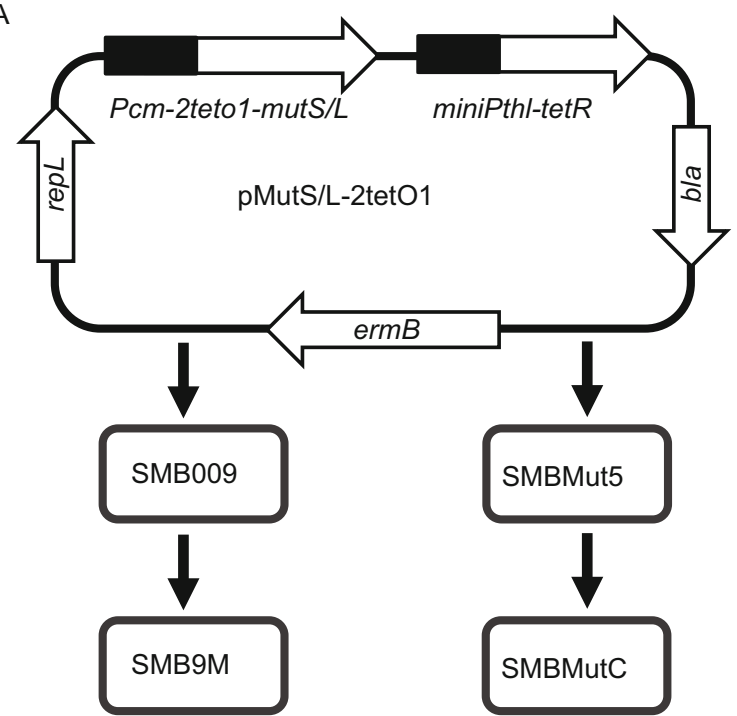

B

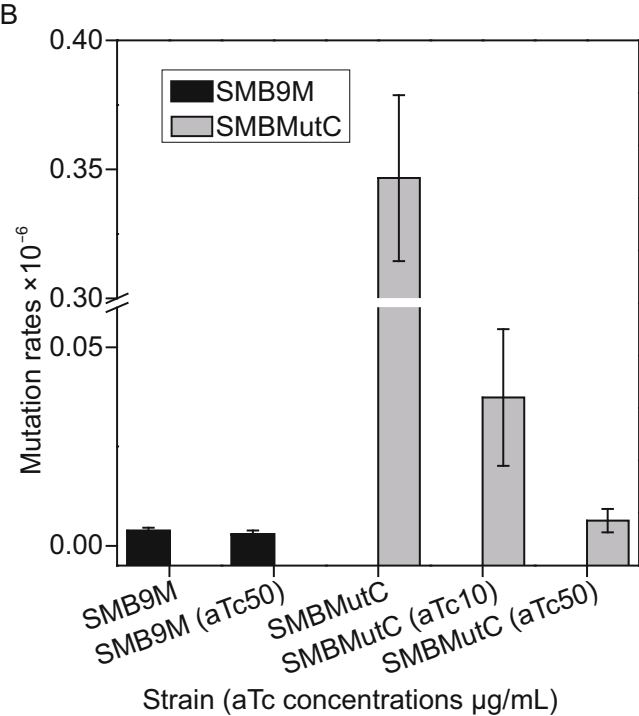

Figure 3. Construction and evaluation of SMBMutC strain. (A) A vector carrying the mutS/L operon under control of an aTc inducible promoter was constructed and termed as pMutS/L-2tetO1. The constructed plasmid was transformed into SMBMut5 and SMB009 to generate SMBMutC strain and SMB9M strain, respectively. (B) Mutation rates determination of SMBMutC strain (grey bars) and SMB9M strain (black bars) under serial concentrations of aTc molecule. Mutation rates were determined after cultivation of the strains for $6 \mathrm{~h}$. Generation frequency of Rif25R colonies was calculated as mutation rates for genome replication. Error bars presented standard deviation among 3 independent determination experiments.

A

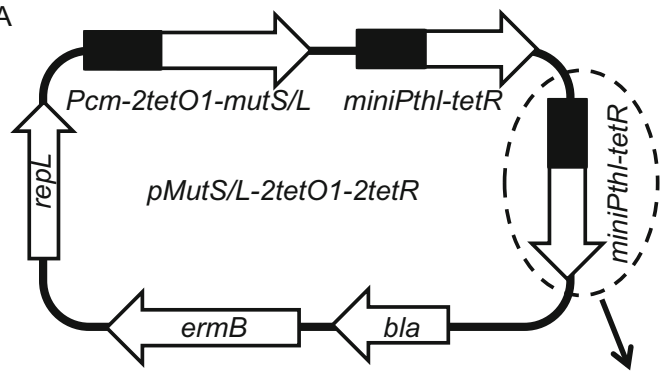

A duplicated miniPthltetR module on pMutS/L-2tetO1-2tetR

B

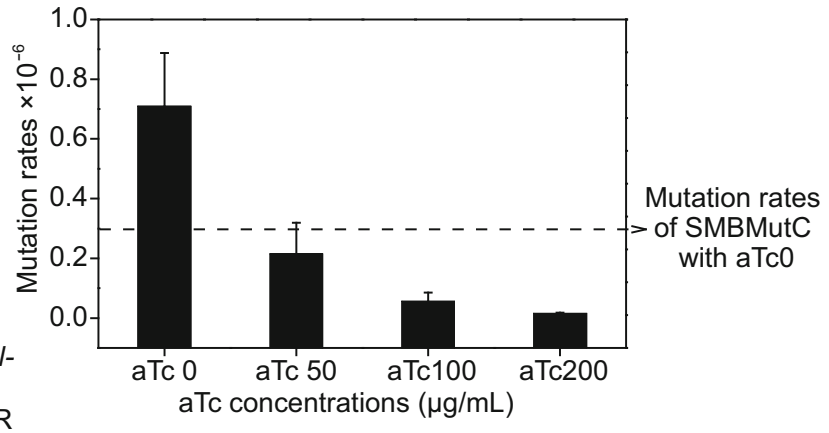

Figure 4. Construction and evaluation of SMBMutC2 strain. (A) miniPthl-tetR component of the pMutS/L-2tetO1 vector was duplicated to generate the pMutS/L-2tetO1-2tetR vector. The obtained vector was transformed into SMBMut5 to generate the SMBMutC2 strain. (B) Mutation rates were determined for SMBMutC2 strain under serial concentrations of aTc. Error bars presented standard deviation among 3 independent determination experiments. The dotted line represented for the mutation rates of SMBMutC with no aTc addition.

SMBMutC2 with elevated mutation rates showed increased survival and tolerance capacities than SMB9M facing butanol-stress

For further evaluating the evolvability and adaptability of the developed controllable hypermutable Clostridium cells, we analyzed and compared the survival and tolerance capacities of the SMBMutC2 and SMB9M under n-butanol challenging conditions. As the main fermentation products of $C$. acetobutylicum, high concentrations of n-butanol are greatly toxic and inhibitory for microbial growths. As designed, SMBMutC2 cells with elevated mutation rates were expected to show enhanced survival and tolerance capacities facing butanol-stress.

After overnight cultivation under normal no-stress conditions, about $10^{6}$ Clostridium cells were spread on RCM agarplates containing series of concentrations of n-butanol and cultivated for 3 days before photographed. As shown in Fig. 5, on non-toxic n-butanol concentration of $10 \mathrm{~g} / \mathrm{L}$, growths of SMBMutC2 and SMB9M showed no difference, and both of them can generate high density lawn on the agar-plates, which were comparable with the growth on normal RCM plates. While on n-butanol concentrations of $12 \mathrm{~g} / \mathrm{L}$, which is toxic for 


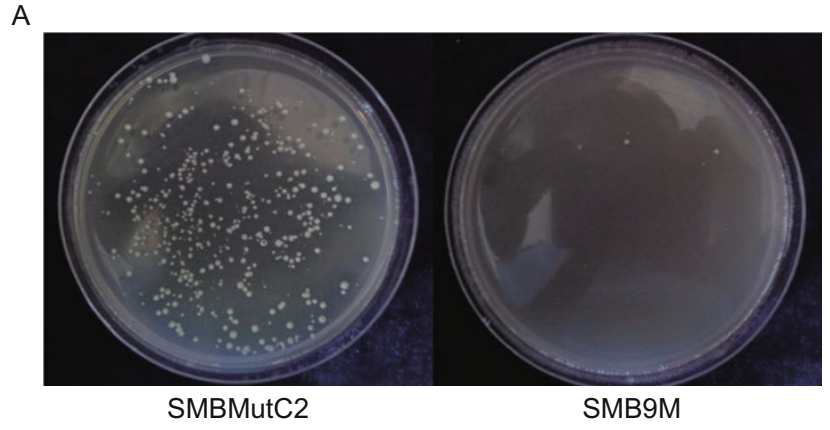

B

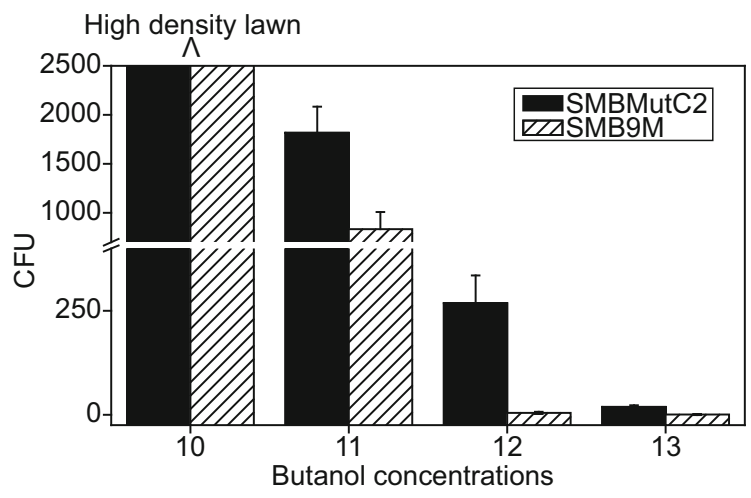

Figure 5. $n$-Butanol challenge of the SMBMutC2 and SMB9M strains. About $10^{6}$ Clostridium cells of SMBMutC2 and SMB9M were spread on RCM agar-plates containing n-butanol concentrations from $10 \mathrm{~g} / \mathrm{L}$ to $14 \mathrm{~g} / \mathrm{L}$, and cultivated for 3 days before photographed and counted. (A) Colonies of SMBMutC2 (left) and SMB9M (right) generated on RCM agar-plates containing $12 \mathrm{~g} / \mathrm{L}$ n-butanol. (B) CFU counts of SMBMutC2 and SMB9M on serial concentrations of $\mathrm{n}$-butanol from $10 \mathrm{~g} / \mathrm{L}$ to $14 \mathrm{~g} / \mathrm{L}$. High density lawn means that the colonies densities were similar with that on n-butanol conditions

cell growths, SMBMutC2 showed greatly increased survival rates than SMB9M (Fig. 5A). Similar phenomena were also observed on other butanol concentrations from $11 \mathrm{~g} / \mathrm{L}$ to $14 \mathrm{~g} / \mathrm{L}$ (Fig. 5B), meaning that the hypermutable Clostridium cells we developed showed increased evolvability and adaptability facing environmental challenges.

\section{DISCUSSION}

Artificial construction of hypermutable cells in which mutation rates were altered via genetic manipulation has been achieved in several microbes. Multiple mutator or anti-mutator genes related with fidelity of DNA replication have been identified in microbes (Horst et al., 1999; Sasaki et al., 2000; Yang et al., 2004), thus providing many targets for genetic manipulation. However, most existing systems for regulating mutation rates were developed by inactivation, over-expression, or modification of a specific mutator or anti-mutator gene, leading to a single-switch control pattern and a constant mutagenesis strength. For better understanding and harnessing microbial evolution, an artificially controllable hypermutable system with fine-tuning capacity might be a better choice (Tenaillon et al., 1999; Loh et al., 2010; Gentile et al., 2011). In this work, we proposed a novel and universal strategy to replace natural cellular controlled expression of MMR system by artificially controlled expression, thus leading to well-controlled mutation rates of microbial genome replication. In C. acetobutylicum, an important and representative Clostridium species, we performed this strategy and constructed an artificially controllable hypermutable system, in which mutation rates of the cells can be well controlled by regulation of aTc concentrations.

Genome sequencing (Bao et al., 2011) and development of efficient genetic manipulation tools (Shao et al., 2007; Dong et al., 2010, 2012) in C. acetobutylicum enabled successful construction of the controllable hypermutable system. mutS and mutL involved in MMR system play an important role for quality control of DNA replication in microbes by recognizing and removing mismatches generated during DNA replication (Modrich and Lahue, 1996; Horst et al., 1999), and their defects have been proved responsible for generation of hypermutable cells in many microbes (LeClerc et al., 1996; Sniegowski et al., 1997; Shaver and Sniegowski, 2003). In this work, inactivation of the chromosome located mutS/L operon and introduction of an aTc-inducible mutS/L expression system successfully constructed an aTc-dose-responsive regulation on the mutation rates of $C$. acetobutylicum. In summary, with the controllable hypermutable strains SMBMutC and SMBMutC2, mutation rates of $C$. acetobutylicum cells can be regulated ranging over 3 orders of magnitudes, from normal level of the wildtype control to 120-fold increased level. Convenient switch between normal cells to hypermutable cells and fine-tuning of the strength of mutation rates indicated this system could act as a powerful tool for harnessing evolution of $C$. acetobutylicum (Hermann et al., 1985; Stephanopoulos, 2002; Liu et al., 2013).

Accuracy of genetic information transfer is guaranteed by multiple and hierarchical mechanisms in microbial cells (Kunkel, 2004), meaning many targets can be manipulated to trigger increased mutation rate for genome replication. Disturbing of different proofreading-related genes would bring influence of varying degrees to fidelity of DNA replication (Morrison et al., 1993; Sasaki et al., 2000). Knockout of the mutS/L operon led to a 250-fold increased mutation rate in C. acetobutylicum, and that is also the theoretically maximal mutagenesis strength that can be achieved in our system. For further expanding the regulatory space to generate higher mutagenesis strengths if needed, systematic modifying and engineering of other proofreading-related genes might be necessary.

The controllable hypermutable system of $C$. acetobutylicum showed improved controllability of genome replication mutation rates, and that confirmed the feasibility and effectiveness of our two-step strategy to replace natural cellular control of the replication-proofreading-mechanism by artificial control. In comparison with the previously developed hypermutable system in other microbes (Selifonova et al., 2001; Shimoda et al., 2006; Abe et al., 2009), the hypermutable cells developed with 
Table 2. Bacterial strains, plasmids and primers

\begin{tabular}{|c|c|c|}
\hline Strains, plasmids, or primers & Relevant Characteristics & Sources \\
\hline \multicolumn{3}{|l|}{ Strains } \\
\hline C. acetobutylicum SMB009 & CAC 1502::intron & Dong et al., 2010 \\
\hline C. acetobutylicum SMB Mut2 & mutS::intron, derived from SMB009 & This work \\
\hline C. acetobutylicum SMB Mut3 & mutL::intron, derived from SMB009 & This work \\
\hline C. acetobutylicum SMB Mut5 & mutS::intron, mutL::intron, derived from SMB009 & This work \\
\hline E. coli JM109 & recA1 $\mathrm{mcrB}^{+}$hsdR17 & Lab storage \\
\hline \multicolumn{3}{|l|}{ Plasmids } \\
\hline pIMP1 & $\mathrm{MLS}^{\mathrm{R}} \mathrm{Amp}^{\mathrm{R}}$ shuttle vector of E. coli-C. acetobutylicum & $\begin{array}{l}\text { Mermelstein et } \\
\text { al., } 1992\end{array}$ \\
\hline pMTL009-mutS & For mutS gene disruption by group II intron method & This work \\
\hline pMTL009-mutL & For mutL gene disruption by group II intron method & This work \\
\hline pGusA2-2tetO1 & aTc inducible $P c m-2$ tetO1-GusA2 expression system & Dong et al., 2012 \\
\hline pMutS/L-2tetO1 & Derived from pGusA2-2tetO1, & This work \\
\hline pMutS/L-2tetO1-2tetR & Derived from pMutS/L-2tetO1, duplicated miniPthl-tetR module & This work \\
\hline \multicolumn{3}{|l|}{ Primers } \\
\hline mutS-570/571S-IBS & AAAAAAGCTTATAATTATCCTTAAGAGAAAGATTTGTGCGCCCAGATAGGGTG & This work \\
\hline mutS-570/571S-EBS1d & CAGATTGTACAAATGTGGTGATAACAGATAAGTCAGATTTGATAACTTACCTTTCTTTG & This work \\
\hline mutS-570/571S-EBS2 & TGAACGCAAGTTTCTAATTTCGGTTTCTCTTCGATAGAGGAAAGTGTCT & This work \\
\hline mutS-disruption-check-F & TCGCATCTAGAGAACTGGAATTAGT & This work \\
\hline mutS-disruption-check-R & AGCACAACTTTTTAATCCTTCTGTCATA & This work \\
\hline mutL-210/211S-IBS & AAAAAAGCTTATAATTATCCTTAGATATAGAAAAAGTGCGCCCAGATAGGGTG & This work \\
\hline mutL-210/211S-EBS1d & CAGATTGTACAAATGTGGTGATAACAGATAAGTCGAAAAAGCTAACTTACCTTTCTTTGT & This work \\
\hline mutL-210/211S-EBS2 & TGAACGCAAGTTTCTAATTTCGGTTATATCTCGATAGAGGAAAGTGTCT & This work \\
\hline mutL-disruption-check-F & ATTGCCGCAGGAGAAGTGGTA & This work \\
\hline mutL-disruption-check-R & AAATCTTCAGGGAGTAATTCCACTA & This work \\
\hline mutS/L-operon-F & ATCGCCATGGTTAGGAGGTTAGTTAGAATGAGCATATCTCCTATGAT & This work \\
\hline mutS/L-operon-R & AAGTTCTCGAGCCCCACAGCTGTAGGTCCTG & This work \\
\hline mini-thl-tetR-F & ATCGCTCGAGTATATTGATAAAAATAATAATAGTGGGTATAATTAAGTTGTTAGGAGG & This work \\
\hline mini-thl-tetR-R & ATCGGGATCCAACTCGACATCTTGGTTACCGTG & This work \\
\hline
\end{tabular}

Abbreviations: $\mathrm{Amp}^{\mathrm{R}}$, ampicillin resistance; $\mathrm{Cm}^{\mathrm{R}}$, chloramphenicol resistance; $\mathrm{MLS}^{\mathrm{R}}$, macrolide, lincosamide, and streptogramin $\mathrm{B}$ resistance.

our method enabled more convenient and regulatable control of cellular mutation rates. Fine-tuning of the mutation rates to diverse levels in our system can be achieved by addition or removal of exogenous signal aTc molecules, rather than complex genetic manipulations. In addition, when desired traits were obtained for the hypermutable cells, the convenient switch from mutational state to normal state will guarantee maintaining of the obtained phenotypes and genotypes by preventing possible back mutations or negative mutations. Based on development of efficient genetic manipulation approaches and genome sequencing technologies, our strategy will encourage and promote development of similar systems in other microbes.

\section{MATERIALS AND METHODS}

Bacterial strains, plasmids, and primers

Bacterial strains, plasmids, and primers used in this work are listed in
Table 2. E. coli DH5a cells (TAKARA) were used for plasmid construction. All primers were synthesized by Invitrogen (Beijing, China) followed by polyacrylamide gel electrophoresis purification.

Cultivation and maintenance conditions

E. coli cells were aerobically cultivated in Luria-Bertani medium at $37^{\circ} \mathrm{C}$ with $200 \mathrm{rpm}$ orbital shaking. All C. acetobutylicum strains were cultivated anaerobically at $37^{\circ} \mathrm{C}$ in RCM medium (Hirsch and Grinsted, 1954). Antibiotics were supplemented when necessary (ampicillin, $100 \mu \mathrm{g} / \mathrm{mL}$, Amp100; chloramphenicol, $30 \mu \mathrm{g} / \mathrm{mL}, \mathrm{Cm} 30$; erythromycin, $50 \mu \mathrm{g} / \mathrm{mL}$, Em50). All E. coli and C. acetobutylicum strains were maintained in $15 \%$ glycerol at $-80^{\circ} \mathrm{C}$.

DNA isolation and manipulation

Total genomic DNA of $C$. acetobutylicum was extracted with TIANamp Bacterial DNA Kit (TIANGEN Biotech, Beijing, China). Plasmid extraction from E. coli, PCR products purification, and gel extraction were 
performed with E.Z.N.A. Plasmid Extraction Kit, E.Z.N.A. Cycle Pure Kit, and E.Z.N.A. Gel Extraction Kit (Omega Biotek Inc., Guangzhou, China), respectively. DNA restriction and cloning were performed according to standard procedures (Sambrook and Russell, 2001). Enzymes used for DNA amplification and manipulation were from NEB (Beijing, China) and used according to the manufacturer's instructions. Electrotransformation of $C$. acetobutylicum was performed as previously described (Mermelstein et al., 1992).

\section{Construction of mutS/L operon disrupted C. acetobutylicum strains}

C. acetobutylicum strain SMB009 capable of accepting unmethylated DNA (Dong et al., 2010) was selected as parental strain for mutS/L operon disruption with ClosTron method previously developed (Shao et al., 2007). Three strains SMBMut2, SMBMut3, and SMBMut5 were constructed for further analysis and investigation, in which mutS, mutL, and both mutS-mutL were disrupted, respectively. Intron insertion sites on mutS and mutL were selected at the position of 570/571 (numbered from the initiator codon of the ORF) on the sense strand (for mutS) and the position of 210/211 on the sense strand (for mutL), and the intron retargeting PCR primers (i.e., mutS-570/571S-IBS, EBS-1d, EBS2) were designed according to a published computer algorithm (Perutka et al., 2004). Construction manipulation for the retargeting vectors and obtaining of the gene-knockout mutants were performed as previously introduced (Dong et al., 2010; Dong et al., 2012). SMBMut5 strain (mutS::intron, mutL::intron) was derived from SMBMut3 (mutL::intron).

\section{Construction of mutS/L expression vector pMutS/L-2tetO1 and pMutS/L-2tetO1-2tetR}

To construct a mutS/L operon expression vector based on the pGusA2-2tetO1 plasmid that we developed previously (Dong et al., 2012), mutS/L operon from the genome of $C$. acetobutylicum SMB009 was amplified with primer mutS/L-operon-F (Ncol) and mutS/L-operon-R (Xhol). The PCR products were used to replace the gusA gene on the pGusA2-2tetO1 plasmid, to which an Ncol site has been introduced in advance. The resulted plasmid was named as pMutS/L-2tetO1.

To improve the stringency of the aTc-inducible mutS/L expression system, the miniPthl-tetR component on the pMutS/L-2tetO1 vector was duplicated. To this end, the miniPthl-tetR component from the pMutS/L-2tetO1 was amplified with primer miniPthl-tetR-F (Xhol) and miniPthl-tetR-R $(B a m \mathrm{HI})$, and the PCR products were inserted into the pMutS/L-2tetO1 between the $\mathrm{Xhol}$ and $\mathrm{BamHI}$ sites to generate the pMutS/L-2tetO1-2tetR vector.

\section{Mutation rates evaluation}

Three single colonies of $C$. acetobutylicum strains were picked up and inoculated into RCM culture medium for overnight activation. The broth was transferred into fresh media with a ratio of 1:100 and cultivated for $6 \mathrm{~h}$ before spreading on RCM agar plates with or without $25 \mu \mathrm{g} / \mathrm{mL}$ of rifamycin. Mutation rates were calculated by dividing the number of rifamycin resistant (Rif25R) colonies by the number of total colonies. To calculate the total number of cells, series of dilutions were performed. Em50 was added to culture mediums when necessary.

\section{n-Butanol challenge}

Single colonies of SMBMutC2 and SMB9M were picked up and inocu- lated into fresh RCM culture medium and cultivated overnight. Broths containing about $10^{6}$ cells were spread on RCM agar-plates containing serial concentrations of $\mathrm{n}$-butanol, from $10 \mathrm{~g} / \mathrm{L}$ to $14 \mathrm{~g} / \mathrm{L}$. The plates were then cultivated anaerobically at $37^{\circ} \mathrm{C}$ for 3 days before photographed. Em50 was also added to the liquid medium and agar-plates.

\section{ACKNOWLEDGEMENTS}

This research was supported by grants from the National Natural Science Foundation of China (Grant No. 31270107), the National Basic Research Program of China (973 Program) (No. 2011CBA00800) and the National High Technology Research and Development Program (863 Program) (No. 2011AA02A208). Yanping Zhang is supported by grants from the Knowledge Innovation Program of the Chinese Academy of Sciences (No. KSCX2-EW-Q-14).

\section{COMPLIANCE WITH ETHICS GUIDELINES}

Guodong Luan, Zhen Cai, Fuyu Gong, Hongjun Dong, Zhao Lin, Yanping Zhang, and Yin Li declare that they have no conflict of interest.

This article does not contain any studies with human or animal subjects performed by the any of the authors.

\section{REFERENCES}

Abe, H., Fujita, Y., Takaoka, Y., Kurita, E., Yano, S., Tanaka, N., and Nakayama, K. (2009). Ethanol-tolerant Saccharomyces cerevisiae strains isolated under selective conditions by over-expression of a proofreading-deficient DNA polymerase delta. J Biosci Bioeng 108, 199-204.

Andreesen, J.B., H.; Gottschalk, G. (1989). Introduction to the physiology and biochemistry of the genus Clostridium. Clostridia, 36.

Bao, G., Wang, R., Zhu, Y., Dong, H., Mao, S., Zhang, Y., Chen, Z., Li, Y., and Ma, Y. (2011). Complete genome sequence of Clostridium acetobutylicum DSM 1731, a solvent-producing strain with multireplicon genome architecture. J Bacteriol 193, 5007-5008.

Bartlett, J.G. (2006). Narrative review: The new epidemic of Clostridium difficile-associated enteric disease. Ann Intern Med 145, 758-764.

Conrad, T.M., Joyce, A.R., Applebee, M.K., Barrett, C.L., Xie, B., Gao, Y., and Palsson, B.O. (2009). Whole-genome resequencing of Escherichia coli K-12 MG1655 undergoing short-term laboratory evolution in lactate minimal media reveals flexible selection of adaptive mutations. Genome Biol 10.

Dong, H., Zhang, Y., Dai, Z., and Li, Y. (2010). Engineering Clostridium strain to accept unmethylated DNA. PLoS One 5, e9038.

Dong, H.J., Tao, W.W., Zhang, Y.P., and Li, Y. (2012). Development of an anhydrotetracycline-inducible gene expression system for solvent-producing Clostridium acetobutylicum: $A$ useful tool for strain engineering. Metab Eng 14, 59-67.

Echols, H., and Goodman, M.F. (1991). Fidelity mechanisms in DNA replication. Annu Rev Biochem 60, 477-511.

Emlyn-Jones, D., Price, G.D., and Andrews, T.J. (2003). Nitrogenregulated hypermutator strain of Synechococcus $\mathrm{sp}$. for use in in vivo artificial evolution. Appl Environ Microbiol 69, 6427-6433.

Endo, A., Sasaki, M., Maruyama, A., and Kurusu, Y. (2006). Temperature adaptation of Bacillus subtilis by chromosomal groEL replacement. Biosci Biotechnol Biochem 70, 2357-2362.

Gentile, C.F., Yu, S.C., Serrano, S.A., Gerrish, P.J., and Sniegowski, P.D. (2011). Competition between high- and higher-mutating strains 
of Escherichia coli. Biol Lett 7, 422-424.

Greener, A., Callahan, M., and Jerpseth, B. (1997). An efficient random mutagenesis technique using an E. coli mutator strain. Mol Biotechnol 7, 189-195.

Hastings, P.J., Bull, H.J., Klump, J.R., and Rosenberg, S.M. (2000). Adaptive amplification: An inducible chromosomal instability mechanism. Cell 103, 723-731.

Hermann, M., Fayolle, F., Marchal, R., Podvin, L., Sebald, M., and Vandecasteele, J.P. (1985). Isolation and characterization of butanol-resistant mutants of Clostridium acetobutylicum. Appl Environ Microbiol 50, 1238-1243.

Hirsch, A., and Grinsted, E. (1954). Methods for the growth and enumeration of anaerobic spore-formers from cheese, with observations on the effect of nisin. J Dairy Res 21, 101-110.

Horst, J.P., Wu, T.H., and Marinus, M.G. (1999). Escherichia coli mutator genes. Trends Microbiol 7, 29-36.

Ishii, K., Matsuda, H., Iwasa, Y., and Sasaki, A. (1989). Evolutionarily stable mutation rate in a periodically changing environment. Genetics $121,163-174$

Jones, D.T., and Woods, D.R. (1986). Acetone-butanol fermentation revisited. Microbiol Rev 50, 484-524.

Kennedy, C.L., Krejany, E.O., Young, L.F., O'Connor, J.R., Awad, M.M., Boyd, R.L., Emmins, J.J., Lyras, D., and Rood, J.I. (2005). The alpha-toxin of Clostridium septicum is essential for virulence. Mol Microbiol 57, 1357-1366.

Kimura, M. (1967). On the evolutionary adjustment of spontaneous mutation rates. Genet Res 9, 23-34.

Kondrashov, A.S. (1995). Modifiers of mutation-selection balance: general approach and the evolution of mutation rates. Genet Res 66, 53-69.

Kunkel, T.A. (2004). DNA replication fidelity. J Biol Chem 279, 1689516898.

Kunkel, T.A., and Bebenek, R. (2000). DNA replication fidelity. Annu Rev Biochem 69, 497-529.

LeClerc, J.E., Li, B., Payne, W.L., and Cebula, T.A. (1996). High mutation frequencies among Escherichia coli and Salmonella pathogens. Science 274, 1208-1211.

Lewis, T.A., Goszczynski, S., Crawford, R.L., Korus, R.A., and Admassu, W. (1996). Products of anaerobic 2,4,6-trinitrotoluene (TNT) transformation by Clostridium bifermentans. Appl Environ Microbiol 62, 4669-4674.

Liu, X.B., Gu, Q.Y., and Yu, X.B. (2013). Repetitive domestication to enhance butanol tolerance and production in Clostridium acetobutylicum through artificial simulation of bio-evolution. Bioresour Technol 130, 638-643.

Loh, E., Salk, J.J., and Loeb, L.A. (2010). Optimization of DNA polymerase mutation rates during bacterial evolution. Proc Natl Acad Sci U S A 107, 1154-1159.

Matic, I., Taddei, F., and Radman, M. (2004). Survival versus maintenance of genetic stability: a conflict of priorities during stress. Res Microbiol 155, 337-341.

Mermelstein, L.D., Welker, N.E., Bennett, G.N., and Papoutsakis, E.T. (1992). Expression of cloned homologous fermentative genes in Clostridium acetobutylicum ATCC 824. Nat Biotechnol 10, 190195.
Modrich, P., and Lahue, R. (1996). Mismatch repair in replication fidelity, genetic recombination, and cancer biology. Annu Rev Biochem $65,101-133$

Morrison, A., Johnson, A.L., Johnston, L.H., and Sugino, A. (1993). Pathway correcting DNA replication errors in Saccharomyces cerevisiae. EMBO J 12, 1467-1473.

Perron, G.G., Hall, A.R., and Buckling, A. (2010). Hypermutability and compensatory adaptation in antibiotic-resistant bacteria. Am Nat 176, 303-311.

Perutka, J., Wang, W., Goerlitz, D., and Lambowitz, A.M. (2004). Use of computer-designed group II introns to disrupt Escherichia coli DExH/D-box protein and DNA helicase genes. J Mol Biol 336, 421-439.

Rehner, S.A., and Samuels, G.J. (1994). Taxonomy and phylogeny of Gliocladium analysed from nuclear large subunit ribosomal DNA sequences. Mycol Res 98, 625-634.

Sakaguchi, G. (1982). Clostridium-Botulinum toxins. Pharmacol Therapeut 19, 165-194.

Sambrook, J., and Russell, D.W. Molecular cloning: A laboratory manual. Cold Spring Harbor Laboratory Press, New York, 2001.

Sasaki, M., Yonemura, Y., and Kurusu, Y. (2000). Genetic analysis of Bacillus subtilis mutator genes. J Gen Appl Microbiol 46, 183-187.

Selifonova, O., Valle, F., and Schellenberger, V. (2001). Rapid evolution of novel traits in microorganisms. Appl Environ Microbiol 67, 3645-3649.

Shao, L., Hu, S., Yang, Y., Gu, Y., Chen, J., Yang, Y., Jiang, W., and Yang, S. (2007). Targeted gene disruption by use of a group II intron (targetron) vector in Clostridium acetobutylicum. Cell Res 17, 963-965.

Shaver, A.C., and Sniegowski, P.D. (2003). Spontaneously arising mutL mutators in evolving Escherichia coli populations are the result of changes in repeat length. J Bacteriol 185, 6076-6082.

Shimoda, C., Itadani, A., Sugino, A., and Furusawa, M. (2006). Isolation of thermotolerant mutants by using proofreading-deficient DNA polymerase delta as an effective mutator in Saccharomyces cerevisiae. Genes Genet Syst 81, 391-397.

Sniegowski, P.D., Gerrish, P.J., and Lenski, R.E. (1997). Evolution of high mutation rates in experimental populations of E. coli. Nature 387, 703-705.

Stephanopoulos, G. (2002). Metabolic engineering by genome shuffling - Two reports on whole-genome shuffling demonstrate the application of combinatorial methods for phenotypic improvement in bacteria. Nat Biotechnol 20, 666-668.

Taddei, F., Radman, M., MaynardSmith, J., Toupance, B., Gouyon, P.H., and Godelle, B. (1997). Role of mutator alleles in adaptive evolution. Nature 387, 700-702.

Tanabe, K., Kondo, T., Onodera, Y., and Furusawa, M. (1999). A conspicuous adaptability to antibiotics in the Escherichia coli mutator strain, dnaQ49. Fems Microbiol Lett 176, 191-196.

Tenaillon, O., Toupance, B., Le Nagard, H., Taddei, F., and Godelle, B. (1999). Mutators, population size, adaptive landscape and the adaptation of asexual populations of bacteria. Genetics 152, 485-493.

Yang, H.J., Wolff, E., Kim, M., Diep, A., and Miller, J.H. (2004). Identification of mutator genes and mutational pathways in Escherichia coli using a multicopy cloning approach. Mol Microbiol 53, 283-295. 